\title{
Soft-gluon resummation effects on parton distributions
}

\author{
Gennaro Corcella ${ }^{1}$ and Lorenzo Magnea ${ }^{2}$ \\ ${ }^{1}$ Department of Physics, CERN, Theory Division, CH-1211 Geneva 23, Switzerland \\ ${ }^{2}$ Dipartimento di Fisica Teorica, Università di Torino and INFN, Sezione di Torino Via P. Giuria 1, I-10125 Torino, Italy
}

(Received 15 September 2005; published 14 October 2005)

\begin{abstract}
We gauge the impact of soft-gluon resummation on quark distributions by performing a simple fit of deep inelastic scattering structure function data using next-to-leading order (NLO) and next-to-leadinglogarithmic- (NLL)-resummed coefficient functions. We make use of NuTeV charged-current data, as well as New Muon Collaboration (NMC) and Bologna-CERN-Dubna-Munich-Saclay (BCDMS) neutralcurrent results, which probe large values of $x$. Our results suggest that the inclusion of resummation effects in global fits of parton distributions is both feasible and desirable, in order to achieve at large $x$ the accuracy goals of the LHC physics program.
\end{abstract}

DOI: 10.1103/PhysRevD.72.074017

PACS numbers: 12.38.Cy, 12.38.Bx, 13.60.-r

\section{INTRODUCTION}

A precise knowledge of parton distribution functions (PDF) in the proton is going to be one of the cornerstones of the physics analysis of LHC data [1], as well as a key ingredient for studies at other high energy accelerators. In hadron collisions, in fact, all high- $p_{\perp}$ final states are produced through the hard scattering of partons, thus both potential new physics signals and standard model backgrounds are affected in shape and normalization by parton distributions.

PDF's are currently determined by several groups [2-4] through fits to different sets of high-energy data. All current fits are performed at least at next-to-leading order (NLO) in perturbative QCD, while the remarkable recent calculation of the three-loop Altarelli-Parisi splitting functions $[5,6]$ has made it possible to perform consistent nextto-next-to-leading order (NNLO) fits, by restricting the data set to cross sections for which the theoretical calculation has been performed to that order.

It is well known, on the other hand, that finite-order QCD calculations are limited in their range of applicability by the occurrence of large logarithms near the boundaries of phase space, both at large and at small values of $x$. These logarithms must be resummed, in order to enlarge the region in which perturbation theory can be trusted. Large- $x$ logarithms, in particular, are known to be related to soft and collinear gluon emission, and their resummation (threshold resummation) is well understood [7,8], and applied to a wide range of hard QCD processes (see, for example, [9]).

In this paper we shall address the possibility of including the effect of threshold resummations in parton fits, and we shall gauge the impact of these effects on large- $x$ quark distributions, by performing a simple analysis of deep inelastic scattering (DIS) data.

We believe that including resummations would be useful in several respects. From a phenomenological viewpoint, making use of resummed predictions would allow for the inclusion of more large- $x$ data points in parton fits. In DIS, for example, data corresponding to values of $W^{2}=$ $Q^{2}(1-x) / x$ smaller than about $15 \mathrm{GeV}^{2}$ are typically excluded from the fits [10], since they cannot be accounted for by making use of NLO perturbative results. Including resummations should lower this bound considerably [11]. Resummations are also known to reduce the theoretical uncertainty of QCD predictions [12], which would correspondingly decrease one of the sources of error for PDF's. Finally, it should be emphasized that, although soft-gluon resummations modify hard cross sections only near partonic threshold, they can affect parton distributions at smaller values of $x$ through sum rules as well as evolution.

Taking a more formal viewpoint, including resummation effects would bring about significant progress in the process of giving a precise definition of a fitted leading twist PDF. Resummations, in fact, are inevitably entangled with power corrections, which become increasingly important near the edges of phase space. This, however, should not be understood as an extra source of ambiguity: on the contrary, the inclusion of resummations highlights an inherent ambiguity, which is always present when finite-order perturbative predictions are used to extract from data the values of operator matrix elements. In general, it is not consistent to attribute a fixed twist to quantities evaluated at finite perturbative orders in a mass-independent regularization scheme, such as dimensional regularization. In such a scheme, one must first give a precise definition of the perturbative contribution to all orders, which entails a definition of power-suppressed contributions. Such a definition can only be given when all-order contributions have been computed, at least in the region of phase space where power corrections are expected to become dominant. This somewhat formal issue could become practical when a sufficiently precise comparison between PDF's obtained by fitting data and PDF's obtained from the lattice becomes possible.

Finally, it should be noted that resummed predictions exist for most of the cross sections used in global PDF fits, although with a varying degree of accuracy. The gold- 
plated process remains inclusive DIS, where, remarkably, we now have a full next-to-next-to-next-to-leading order (NNNLO) QCD prediction [13], as well as next-to-next-toleading logarithmic (NNLL) soft-gluon resummation [14], and refined QCD-motivated models of the leading power corrections [15]; even a class of nonlogarithmic terms has been shown to exponentiate [16]. The Drell-Yan cross section is understood with almost the same degree of accuracy [14,16], with the added feature of a recent NNLO computation of the vector boson rapidity distribution [17]. This is interesting because it has recently been shown [18] that reasonably competitive parton fits can be obtained on the basis of these two processes only. If one wishes to rely upon a wider data set, next-to-leading logarithmic (NLL) resummed predictions exist also for the prompt-photon production cross section [19]; there, however, phenomenological problems remain, partly associated with a possible inconsistency of different data sets, and possibly related to the need to perform a more refined resummation [20] and to include consistently powersuppressed corrections [21]. Jet production in hadron collisions is more problematic: in fact, although the theoretical tools to perform NLL resummation have been available for some time [22] and a phenomenological study has been performed in [23], it has recently been pointed out that, for most jet definitions, jet cross sections are plagued by nonglobal logarithms [24], starting at NLL level. Pushing the accuracy of soft-gluon resummation beyond leading logarithms (LL) for these cross sections will thus require more work.

It seems fair to conclude that enough resummation technology exists to perform a resummed global PDF fit. Including only DIS and Drell-Yan data, such a fit could actually be consistently performed at NNLO/NNLL level; in order to further constrain combinations of partons, which are hard to determine using these data only, one might then decide to trade some logarithmic accuracy in exchange for more data coverage.

In order to assess the impact that the inclusion of resummations might have on parton distributions, and more specifically on large- $x$ quark distributions, in the following we shall perform a fit of large- $x$ DIS data, using both NLO and NLL-resummed coefficient functions. It should not be regarded as an attempt to a global fit (see, e.g., Ref. [25] for an analysis of large- $x$ PDF's in the context of a global fit), since we shall clearly be forced to make several approximations in order to extract partons from such a comparatively small data set. Rather, it should be seen as a toy model of a resummed fit, providing a rough quantitative assessment of the impact of resummations. We find that soft-gluon effects typically suppress quark distributions by amounts ranging from a few percent to about $15-20 \%$ at large but not extreme values of $x, 0.55 \leq x \leq 0.75$, for moderate $Q^{2}$. Sum rules also force a compensating enhancement in the distribution at smaller values of $x$, which, however, cannot be reliably determined within our current approximations. These effects would indeed warrant a more detailed investigation, if the current goal for PDFrelated uncertainties (a few percent) were to be enforced also at these relatively large values of $x$.

\section{DATA AND PARAMETRIZATIONS}

Large- $x$ DIS data come predominantly from fixed-target experiments. In order to have at our disposal different linear combinations of large- $x$ partons, we shall consider here charged-current (CC) data from neutrino-iron DIS, collected by the NuTeV Collaboration [26,27], and neutralcurrent (NC) data from muon scattering from the NMC [28] and BCDMS [29,30] Collaborations.

For our purposes, it will be sufficient to examine data at fixed values of $Q^{2}$, which we shall pick not too small so as to minimize the impact of power corrections, which are enhanced at the boundaries of phase space. We also require good data coverage for all the three experiments considered. We shall use $Q^{2}=31.62 \mathrm{GeV}^{2}$ and $Q^{2}=$ $12.59 \mathrm{GeV}^{2}$, which corresponds to a cut in $W^{2}$ between 4 and $5 \mathrm{GeV}^{2}$, given the measured values of $x$. We shall check at the end that our results at the two selected values of $Q^{2}$ are compatible with NLO perturbative evolution. Since threshold resummation naturally takes place in Mellin moment space, our procedure will be to construct parametrizations of the data at the chosen values of $Q^{2}$, compute Mellin moments of the parametrizations, and then use them to extract moments of the corresponding PDF's, with and without resummation. The difference between resummed and unresummed moments of PDF's is per se a useful and solid result, since any QCD analysis can in principle be reformulated in Mellin space. In any case, we will also provide a simple $x$-space parametrization in order to illustrate the impact of the results in a more conventional manner. Studies of DIS structure functions in moment space were also performed in [31], by making use of data from the CLAS detector at Jefferson Laboratory; the corresponding values of $Q^{2}$ are however too small for a perturbative study like the present one.

Let us now turn to the NMC, BCDMS and $\mathrm{NuTeV}$ data sets we are considering. An efficient and convenient parametrization of NMC and BCDMS data for the NC structure function $F_{2}$, for proton, deuteron, and separately for the nonsinglet combination, has been provided in Ref. [32], and was recently upgraded for protons with the inclusion of HERA data in Ref. [33]. The parametrization was constructed by first generating a large set of Monte Carlo copies of the original data, including all information on errors and correlations; subsequently, a neural network was trained on each copy of the data, yielding a set of parametrizations which, taken together, give a faithful and unbiased representation of the probability distribution in the space of structure functions. 
In principle, the neural parametrization can be used for any values of $x$ and $Q^{2}$. In practice, errors will become increasingly large when one moves away from the region of the data. We use values of $Q^{2}$ which are well inside the measured region, with data coverage up to $x=0.75$. Specifically, we will be interested in the nonsinglet structure function $F_{2}^{\mathrm{ns}}\left(x, Q^{2}\right)$, which is unaffected by the gluon contribution and provides a combination of quark distributions, essentially $u-d$, which is linearly independent from the ones sampled by NuTeV data. The neural parametrization of $F_{2}^{\mathrm{ns}}\left(x, Q^{2}\right)$ was previously used [34] in conjunction with the technique of truncated Mellin moments [35] for a determination of $\alpha_{s}$, which is unaffected by parametrization biases.

To illustrate the quality of the data, we show in Fig. 1 the nonsinglet structure function $F_{2}^{\text {ns }}\left(x, Q^{2}\right)$, computed with the neural parametrization at our chosen values of $Q^{2}$, and for $x=n / 40, n=1, \ldots, 39$. The central values are given by the averages of the results obtained with the 1000 neural networks of the NNPDF Collaboration, and error bars are the corresponding standard deviations. Error bars are relatively large, because $F_{2}^{\text {ns }}\left(x, Q^{2}\right)$ is the difference between proton and deuteron structure functions, which entails a loss of precision. Central values and errors for the moments are similarly obtained by computing the moments with each neural network, and then taking averages and standard deviations.

$\mathrm{NuTeV}$ provides data for the CC structure functions $F_{2}$ and $F_{3}$. Since data are taken on an iron target, they need to be rescaled to include nuclear corrections, which were computed in [36] by fitting the ratio $F_{2}^{F e} / F_{2}^{D}$. The required smearing factor is given by

$$
N(x)=1.10-0.36 x-0.28 \exp (-21.94 x)+2.77 x^{14.41} .
$$

We consider first the charged-current structure function $F_{3}$ and its parton content. One has

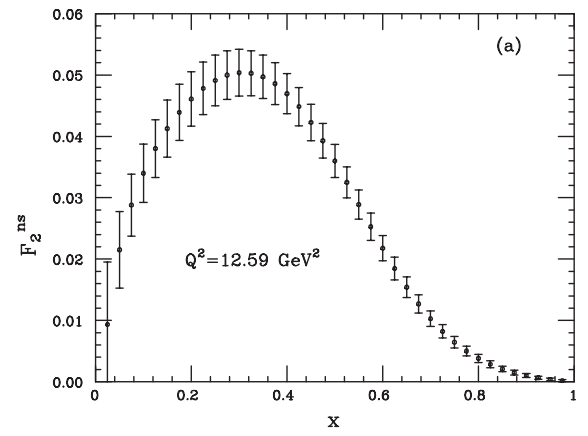

$$
x F_{3}=\frac{1}{2}\left(x F_{3}^{\nu}+x F_{3}^{\bar{\nu}}\right)=x\left[\sum_{q, q^{\prime}}\left|V_{q q^{\prime}}\right|^{2}(q-\bar{q}) \otimes C_{3}^{q}\right],
$$

where $V_{q q^{\prime}}$ are the relevant Cabibbo-Kobayashi-Maskawa (CKM) matrix elements and $C_{3}^{q}$ is the appropriate coefficient function. We fit the data at our chosen values of $Q^{2}$ using the functional form

$$
x F_{3}(x)=C x^{-\rho}(1-x)^{\sigma}(1+k x) .
$$

Equation (2.3) is quite similar to the functional form which is used as initial condition for parton densities in the global analyses [2,3]. We checked the stability of our fit by modifying the last factor of Eq. (2.3) with the inclusion of further powers of $x$ or logarithmic terms in $x$. We find that the parametrization (2.3), with four tunable parameters, is reliable enough to reproduce the data with quite small errors on the best-fit parameters and reasonable values of the $\chi^{2}$ per degree of freedom.

The best-fit values at $Q^{2}=31.62 \mathrm{GeV}^{2}$ are $C=$ $0.103 \pm 0.012, \rho=0.294 \pm 0.034, \sigma=3.325 \pm 0.089$, $k=42.972 \pm 4.700$, corresponding to $\chi^{2} / \mathrm{dof}=7.20 / 6$. At $Q^{2}=12.59 \mathrm{GeV}^{2}$ we find instead $C=0.054 \pm 0.005$, $\rho=0.245 \pm 0.038, \quad \sigma=3.374 \pm 0.145, \quad k=99.719 \pm$ 0.247 , corresponding to $\chi^{2} / \mathrm{dof}=2.06 / 6$. The data and the best-fit curves at the relevant values of $Q^{2}$ are shown in Fig. 2.

The situation for the structure function $F_{2}$, extracted from charged-current data, is slightly more complicated since there is a singlet component, and thus gluon-initiated processes also contribute. Such processes are not logarithmically enhanced at large $x$, and in fact, in the region of interest for our purposes, the gluon contribution to the structure function is significantly suppressed. We will handle it by subtracting it from the data point by point, using a gluon distribution determined by a global fit. The parton content of the charged-current structure function $F_{2}$ is

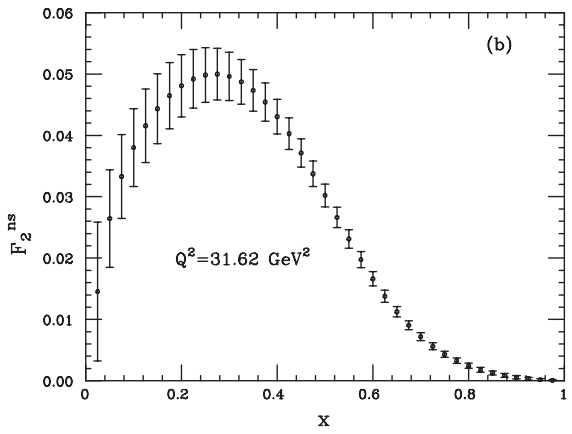

FIG. 1. A sampling of the neural parametrization of NMC and BCDMS data for $F_{2}^{\mathrm{ns}}\left(x, Q^{2}\right)$ at $Q^{2}=12.59 \mathrm{GeV}^{2}$ (a) and at $Q^{2}=$ $31.62 \mathrm{GeV}^{2}$ (b), from the NNPDF Collaboration [32]. 

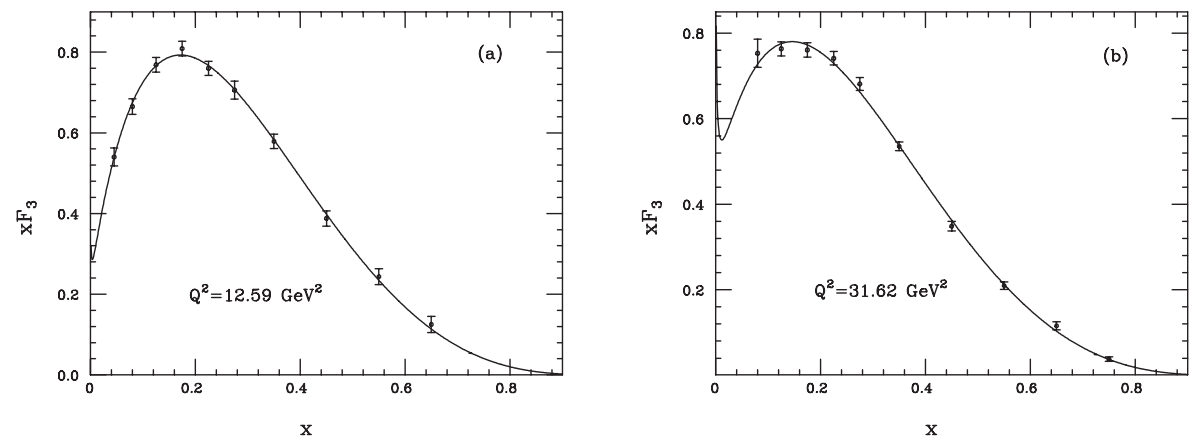

FIG. 2. NuTeV data on the structure function $x F_{3}$, at $Q^{2}=12.59 \mathrm{GeV}^{2}$ (a) and at $Q^{2}=31.62 \mathrm{GeV}^{2}$ (b), along with the best-fit curve parametrized by Eq. (2.3).

$$
\begin{aligned}
F_{2} & \equiv \frac{1}{2}\left(F_{2}^{\nu}+F_{2}^{\bar{\nu}}\right)=x \sum_{q, q^{\prime}}\left|V_{q q^{\prime}}\right|^{2}\left[(q+\bar{q}) \otimes C_{2}^{q}+g \otimes C_{2}^{g}\right] \\
& =F_{2}^{q}+F_{2}^{g} .
\end{aligned}
$$

We will proceed by fitting only $F_{2}^{q}$ and computing the gluon-initiated contribution using the gluon distribution from the NLO set CTEQ6M [2]. We have checked that our results are not affected by the specific choice of gluon density, by repeating the calculation with, e.g., the set MRST2001 [3]. As above, we pick the parametrization

$$
F_{2}^{q}(x)=F_{2}(x)-F_{2}^{g}(x)=A x^{-\alpha}(1-x)^{\beta}(1+b x) .
$$

When doing the fit, we assume that we can neglect correlations among data points, as well as the error on $F_{2}^{g}$ with respect to the error on $F_{2}$ quoted by $\mathrm{NuTeV}$. At $Q^{2}=$ $31.62 \mathrm{GeV}^{2}$, the best-fit values for the parameters in Eq. (2.5) are $A=0.240 \pm 0.002, \alpha=0.562 \pm 0.020, \beta=$ $3.211 \pm 0.065, \quad b=13.085 \pm 0.767, \quad$ with $\quad \chi^{2} /$ dof $=$ 9.99/6. At $Q^{2}=12.59 \mathrm{GeV}^{2}$, on the other hand, we find $A=0.038 \pm 0.005, \quad \alpha=0.816 \pm 0.021, \quad \beta=2.697 \pm$ $0.050, b=66.804 \pm 7.583$, with $\chi^{2} /$ dof $=9.55 / 6$. In Fig. 3 we plot the data points of $F_{2}^{q}(x)$ at the chosen values of $Q^{2}$, along with the curve given by Eq. (2.5), according to the central values of the best-fit parameters.

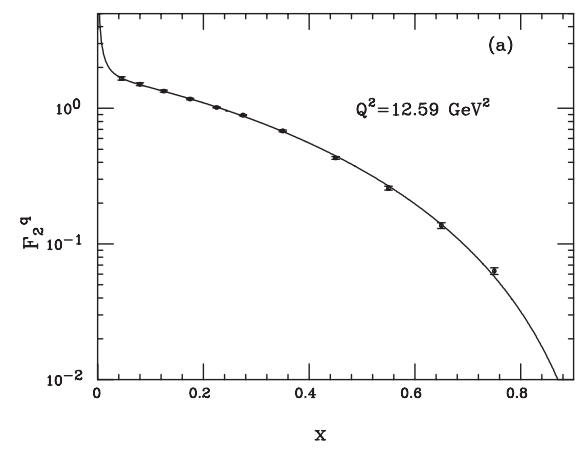

At this point we have at our disposal parametrized expressions, including errors and correlations for the parameters, within the stated approximations, for the structure functions $x F_{3}, F_{2}^{q}$, and $F_{2}^{\text {ns }}$. We can thus compute moments for the specified values of $Q^{2}$, and extract the moments of the corresponding parton densities by dividing out the appropriate coefficient functions, with and without resummations.

\section{A SIMPLE PARTON FIT}

Having subtracted the contribution of gluon-initiated processes from the charged-current structure function $F_{2}$ in Eq. (2.4), the factorization

$$
F_{i}\left(x, Q^{2}\right)=x \int_{x}^{1} \frac{d \xi}{\xi} q_{i}\left(\xi, \mu_{F}^{2}\right) C_{i}\left(\frac{x}{\xi}, \frac{Q^{2}}{\mu_{F}^{2}}, \alpha_{s}\left(\mu_{R}^{2}\right)\right)
$$

applies to all the structure functions we shall be considering $\left(F_{i}=\left\{F_{2}, x F_{3}, F_{2}^{\text {ns }}\right\}\right)$. In all cases $q_{i}$ is a combination of (anti)quark distributions, while $C_{i}$ is the appropriate coefficient function.

The coefficient functions $C_{i}$ for quark-initiated DIS contain terms that become large when the Bjorken variable $x$ for the partonic process is close to $x=1$, which forces gluon radiation from the incoming quark to be soft or collinear. At $\mathcal{O}\left(\alpha_{s}\right)$, for example, the coefficient functions

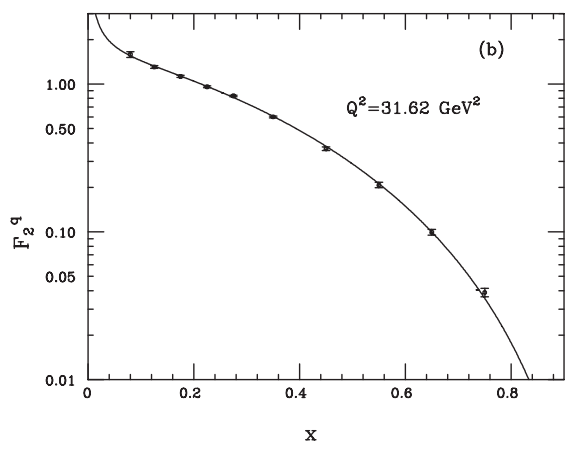

FIG. 3. NuTeV data on the quark-initiated contribution $F_{2}^{q}$ to the structure function $F_{2}$, for $Q^{2}=12.59 \mathrm{GeV}^{2}$ (a), and $Q^{2}=$ $31.62 \mathrm{GeV}^{2}$ (b). The solid lines are the best-fit predictions according to Eq. (2.5). 
can be written in the form

$$
C_{i}^{\mathrm{NLO}}\left(x, \frac{Q^{2}}{\mu_{F}^{2}}, \alpha_{s}\left(\mu_{R}^{2}\right)\right)=\delta(1-x)+\frac{\alpha_{s}\left(\mu_{R}^{2}\right)}{2 \pi} H_{i}\left(x, \frac{Q^{2}}{\mu_{F}^{2}}\right) .
$$

Treating all quarks as massless, the part of $H_{i}$ which contains terms that are logarithmically enhanced as $x \rightarrow$ 1 reads

$$
\begin{aligned}
H_{i, \mathrm{soft}}\left(x, \frac{Q^{2}}{\mu_{F}^{2}}\right)= & 2 C_{F}\left\{\left[\frac{\ln (1-x)}{1-x}\right]_{+}\right. \\
& \left.+\frac{1}{(1-x)_{+}}\left(\ln \frac{Q^{2}}{\mu_{F}^{2}}-\frac{3}{4}\right)\right\} .
\end{aligned}
$$

Taking a Mellin transform, the contributions proportional to $\alpha_{s}[\ln (1-x) /(1-x)]_{+}$and to $\alpha_{s}[1 /(1-x)]_{+}$correspond to double $\left(\alpha_{s} \ln ^{2} N\right)$ and single $\left(\alpha_{s} \ln N\right)$ logarithms of the Mellin variable $N$. Retaining only terms that are singular at large $N$ one finds in fact

$$
\hat{H}_{i, \text { soft }}\left(N, \frac{Q^{2}}{\mu_{F}^{2}}\right)=2 C_{F}\left\{\frac{1}{2} \ln ^{2} N+\left[\gamma_{E}+\frac{3}{4}-\ln \frac{Q^{2}}{\mu_{F}^{2}}\right] \ln N\right\} \text {. }
$$

The resummation of soft-gluon effects, responsible for this singular behavior of DIS structure functions, has been well understood for a long time [7,8]: it results in exponentiation of all singular contributions to the Mellin moments of the coefficient functions $C_{i}$ at large values of the moment variable $N$. In the $\overline{\mathrm{MS}}$ factorization scheme, soft resummation was implemented in $[14,37]$ in the massless approximation, and in [38,39] for heavy quark production. In the following, we shall consider values of $Q^{2}$ much larger than the relevant quark masses, so that we can safely apply the results in the massless approximation.

The pattern of exponentiation of logarithmic singularities is nontrivial: one finds that Mellin moments of the coefficient functions can be written as

$$
\begin{aligned}
\hat{C}_{i}^{\text {res }}\left(N, \frac{Q^{2}}{\mu_{F}^{2}}, \alpha_{s}\left(\mu_{R}^{2}\right)\right)= & \mathcal{R}\left(N, \frac{Q^{2}}{\mu_{F}^{2}}, \alpha_{s}\left(\mu_{R}^{2}\right)\right) \\
& \times \Delta\left(N, \frac{Q^{2}}{\mu_{F}^{2}}, \alpha_{s}\left(\mu_{R}^{2}\right)\right),
\end{aligned}
$$

where $\mathcal{R}$ is a finite remainder, nonsingular as $N \rightarrow \infty$, while $[7,8,40]$

$$
\begin{aligned}
\ln \Delta\left(N, \frac{Q^{2}}{\mu_{F}^{2}}, \alpha_{s}\left(\mu_{R}^{2}\right)\right)= & \int_{0}^{1} d x \frac{x^{N-1}-1}{1-x} \\
& \times\left\{\int_{\mu_{F}^{2}}^{(1-x) Q^{2}} \frac{d k^{2}}{k^{2}} A\left[\alpha_{s}\left(k^{2}\right)\right]\right. \\
& \left.+B\left[\alpha_{s}\left(Q^{2}(1-x)\right)\right]\right\} .
\end{aligned}
$$

In Eq. (3.6) the leading logarithms (LL), of the form $\alpha_{s}^{n} \ln ^{n+1} N$, are generated at each order by the function $A$. Next-to-leading logarithms (NLL), on the other hand, of the form $\alpha_{s}^{n} \ln ^{n} N$, require the knowledge of the function $B$. In general, resumming $\mathrm{N}^{k} \mathrm{LL}$ to all orders requires the knowledge of the function $A$ to $k+1$ loops, and of the function $B$ to $k$ loops. In the following, we will adopt the common standard of NLL resummation, therefore we need the expansions

$$
A\left(\alpha_{s}\right)=\sum_{n=1}^{\infty}\left(\frac{\alpha_{s}}{\pi}\right)^{n} A^{(n)} ; \quad B\left(\alpha_{s}\right)=\sum_{n=1}^{\infty}\left(\frac{\alpha_{s}}{\pi}\right)^{n} B^{(n)}
$$

to second order for $A$ and to first order for $B$. The relevant coefficients are

$$
\begin{gathered}
A^{(1)}=C_{F}, \quad A^{(2)}=\frac{1}{2} C_{F}\left[C_{A}\left(\frac{67}{18}-\frac{\pi^{2}}{6}\right)-\frac{5}{9} n_{f}\right], \\
B^{(1)}=-\frac{3}{4} C_{F} .
\end{gathered}
$$

Notice that in Eq. (3.6) the term containing the function $A\left(\alpha_{s}\right)$ resums the contributions of gluons that are both soft and collinear, and in fact the anomalous dimension $A$ can be extracted order by order from the residue of the singularity of the nonsinglet splitting function as $x \rightarrow 1$. The function $B$, on the other hand, is related to collinear emission from the final state current jet. In the case of heavy quarks, the function $B\left(\alpha_{s}\right)$ needs to be replaced by a different function, called $S\left(\alpha_{s}\right)$ in [38], which is instead characteristic of processes with massive quarks, and includes effects of large-angle soft radiation.

Turning to our fit, we observe that, upon taking Mellin moments, the convolution in Eq. (3.1) turns into a product, and it becomes straightforward to extract moments of the parton combinations $q_{i}\left(x, Q^{2}\right)$ at NLO, or with NLL resummation. Setting $\mu_{F}=\mu_{R}=Q$, one simply finds

$$
\begin{aligned}
\hat{q}_{i}^{\mathrm{NLO}}\left(N, Q^{2}\right) & =\frac{\hat{F}_{i}\left(N-1, Q^{2}\right)}{\hat{C}_{i}^{\mathrm{NLO}}\left(N, 1, \alpha_{s}\left(Q^{2}\right)\right)} ; \\
\hat{q}_{i}^{\mathrm{res}}\left(N, Q^{2}\right) & =\frac{\hat{F}_{i}\left(N-1, Q^{2}\right)}{\hat{C}_{i}^{\mathrm{res}}\left(N, 1, \alpha_{s}\left(Q^{2}\right)\right)},
\end{aligned}
$$

where the resummed coefficient function has been suitably matched to NLO, in order to avoid double counting of logarithmic contributions.

Since we are considering only three measurements, we need to introduce further approximations in order to be able to extract individual parton distributions. We will use isospin symmetry of the sea, so that $\bar{u}=\bar{d}$ and $s=\bar{s}$; further, we shall take the charm quark distribution to vanish and, for simplicity, we will impose a simple proportionality relation between antiquark distributions, $\bar{s}=\kappa \bar{u}$. In the fit shown below, we shall assume $\kappa=1 / 2$. All of these assumptions are essentially harmless at large $x$, where sea quarks are negligible: they allow us, however, to solve 
for the valence quark distributions $u, d$ and, say, $s$. The expressions for the parton combinations $q_{i}$ become particularly simple if one approximates the elements of the CKM matrix by neglecting terms of order $\left(\sin \theta_{C}\right)^{4}$ in $\left|V_{q q^{\prime}}\right|^{2}$. Within the stated approximations, one finds then

$$
\begin{aligned}
& q_{2}\left(x, Q^{2}\right)=u\left(x, Q^{2}\right)+d\left(x, Q^{2}\right)+3 s\left(x, Q^{2}\right), \\
& q_{3}\left(x, Q^{2}\right)=u\left(x, Q^{2}\right)+d\left(x, Q^{2}\right)-s\left(x, Q^{2}\right), \\
& q_{2}^{\mathrm{ns}}\left(x, Q^{2}\right)=\frac{1}{6}\left(u\left(x, Q^{2}\right)-d\left(x, Q^{2}\right)\right),
\end{aligned}
$$

which is easily inverted to give $u, d$, and $s$. Having extracted the moments of the parton combinations in Eq. (3.10), one easily derives moments of individual quark distributions. In order to estimate the error on the moments, we proceed as follows. Regarding $F_{2}^{\text {ns }}$, the neural parametrization is designed to allow for a simple calculation of the error on any functional of the data: one simply computes the standard deviation of the desired observable over the set of neural nets. A similar procedure would also yield correlations between different observables (in our case moments). In the present case, we shall neglect correlations between moments since we do not have a sufficiently reliable method to evaluate them in the case of $\mathrm{NuTeV}$ data. Concerning charged-current structure functions, we mimick the neural method by generating a Monte Carlo set of parametrizations of $F_{2}$ and $F_{3}$ at the chosen values of $Q^{2}$, assuming the parameters of our fits are Gaussian distributed around their mean values with the stated errors. We then compute errors on moments as standard deviations over the Monte Carlo set.

Our results for the moments of the valence up quark distribution are shown in Figs. 4 and 5. The trend is clear, as could have been anticipated by comparing the resummed coefficient function with the NLO one: in the $\overline{\mathrm{MS}}$ scheme, resummation enhances the moments of the

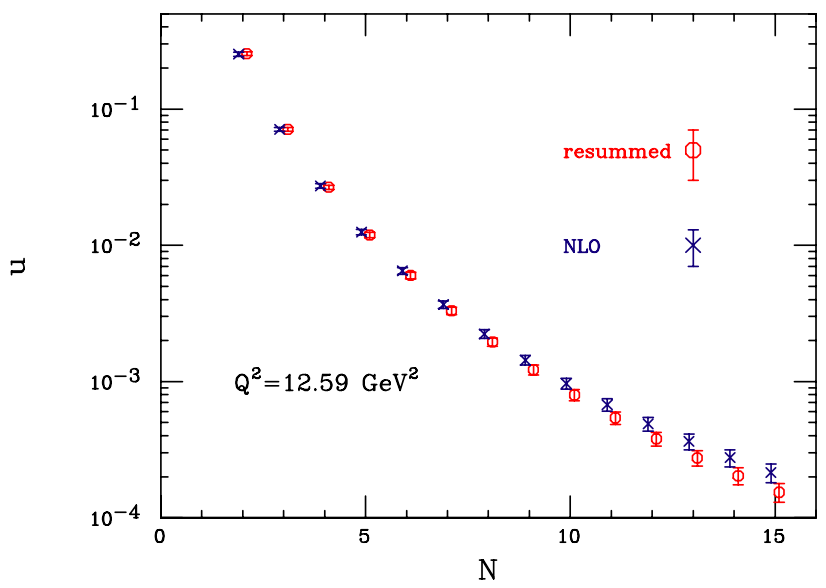

FIG. 4 (color online). Moments of the resummed and NLO up quark distribution with statistical errors, in the range $2 \leq N \leq$ 15 , at $Q^{2}=12.59 \mathrm{GeV}^{2}$.

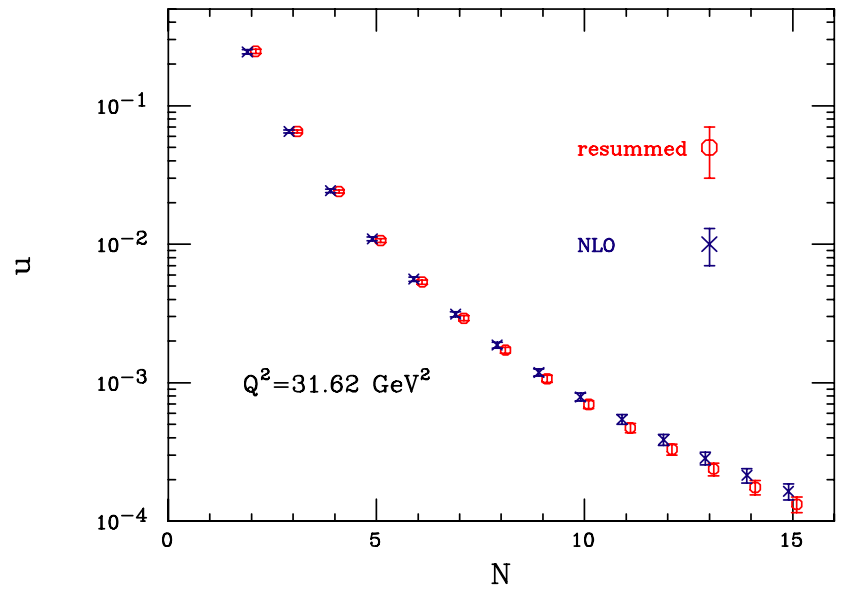

FIG. 5 (color online). As in Fig. 4, but at $Q^{2}=31.62 \mathrm{GeV}^{2}$.

coefficient function, and thus suppresses the moments of quark distributions, with an effect increasing with the moment index $N$. The effect is unequivocal at both values of $Q^{2}$, since resummed and NLO moments differ by more than their error, beginning with $N \sim 6$. At $Q^{2}=$ $31.62 \mathrm{GeV}^{2}$ errors are somewhat smaller, however the effect of resummation is also slightly reduced, so that errors tend to have a somewhat larger overlap. In fact, as observed, e.g., in Ref. [38], LL and NLL terms in the Sudakov exponent are weighted by powers of $\alpha_{s}$ and the coupling constant is larger when evaluated at a lower scale. The trend is, in any case, still clearly visible, and the shift in the central values is of comparable size.

The down quark distribution is significantly smaller than the up quark distribution at large $x$, thus large- $N$ moments are suppressed. With our current, relatively small data set, the effect of resummation on the down quark distribution is completely overshadowed by statistical errors, so that the values of the moments are compatible with 0 beginning at $N \sim 10$. Similarly, moments of the strange quark distribution cannot be reliably determined with these data. We shall then concentrate on the up quark distribution for the remainder of our analysis. One may just note in passing that resummation does not appear to shift even the central values of the moments of $d\left(x, Q^{2}\right)$ : taken seriously, this would suggest that the bulk of the effect is carried by the largest valence distribution for the chosen hadron.

In order to provide a more conventional (and possibly a more practical) parametrization of the effect of resummations, we have also performed a fit of the moments presented in Figs. 4 and 5 to a simple $x$-space parametrization, choosing

$$
u(x)=D x^{-\gamma}(1-x)^{\delta} .
$$

The functional form (3.11) is sufficient to fit the moments of the up quark distribution with small errors on $D, \gamma$, and $\delta$, and a low $\chi^{2} /$ dof. We have checked that the inclusion of other terms, for example, a further factor linear in $x$, 


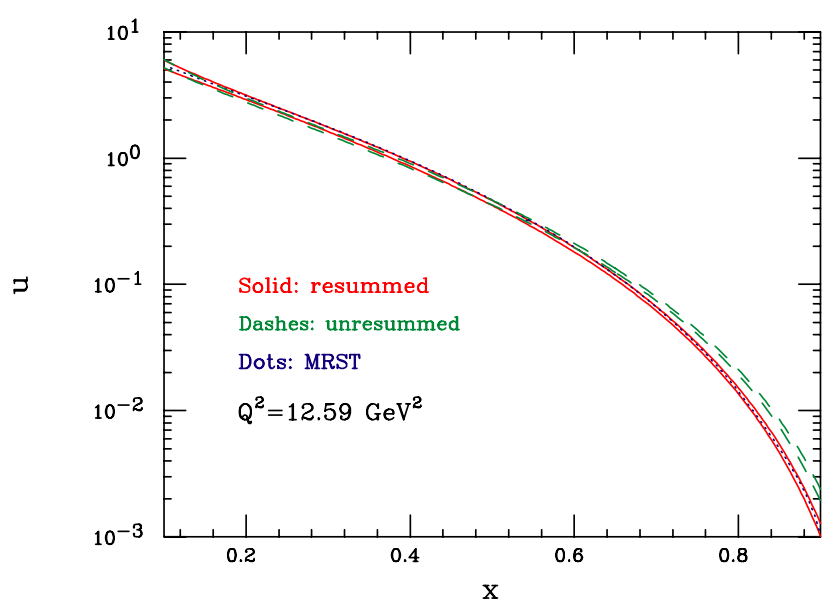

FIG. 6 (color online). Resummed and NLO up quark distributions, at $Q^{2}=12.59 \mathrm{GeV}^{2}$, reconstructed by fitting moments with a simple functional form, $u(x)=D x^{-\gamma}(1-x)^{\delta}$. The MRST2001 NLO up quark distribution at the appropriate $Q^{2}$ is included for comparison. The plot shows the edges of a band at the one-standard-deviation confidence level.

as in Eqs. (2.3) and (2.5) does not significantly improve the quality of the fit. The results using the fitting function (3.11) are shown in Figs. 6 and 7. The best-fit parameters at $Q^{2}=12.59 \mathrm{GeV}^{2}$ are $D=3.025 \pm 0.534, \gamma=0.418 \pm$ 0.101 , and $\delta=3.162 \pm 0.116$, with $\chi^{2} /$ dof $=1.62 / 11$, for the NLO fit, while the resummed fit yields $D=$ $4.647 \pm 0.881, \quad \gamma=0.247 \pm 0.109, \quad$ and $\delta=3.614 \pm$ 0.128 , with $\chi^{2} /$ dof $=0.64 / 11$. At $Q^{2}=31.62 \mathrm{GeV}^{2}$ we find $D=2.865 \pm 0.420, \quad \gamma=0.463 \pm 0.086, \quad \delta=$ $3.301 \pm 0.098$, and $\chi^{2} /$ dof $=1.10 / 11$ for the NLO fit, as well as $D=3.794 \pm 0.583, \gamma=0.351 \pm 0.090, \delta=$ $3.598 \pm 0.104$ and $\chi^{2} /$ dof $=0.53 / 11$ for the resummed fit $^{1}$. The error bands in Figs. 6 and 7 correspond to a prediction at one-standard-deviation confidence level. They are obtained, as above, by generating a Monte Carlo sample of parametrizations of the stated form, assuming a Gaussian distribution for the parameters $D, \gamma$, and $\delta$ with the stated errors; thus, they reflect only statistical errors and do not take into account biases due to the simple choice of functional form.

To display more clearly the quantitative effect of the resummation, we also present in Fig. 8 the central values for the normalized deviation of the resummed prediction from the NLO distribution, $\Delta u(x)=\left(u_{\mathrm{NLO}}(x)-\right.$ $\left.u_{\text {res }}(x)\right) / u_{\mathrm{NLO}}(x)$, at the two chosen values of $Q^{2}$.

A few comments concerning Figs. 6-8 are in order. First of all, an evident feature of the result is the change in sign of the effect around the point $x=1 / 2$. This is a stable feature of all our fits, and it can be traced back to the momentum sum rule, which is essentially unaffected by the

\footnotetext{
${ }^{1}$ We note that the values of $\chi^{2} /$ dof are very small. This might be due to the fact that we are neglecting correlations between moments.
}

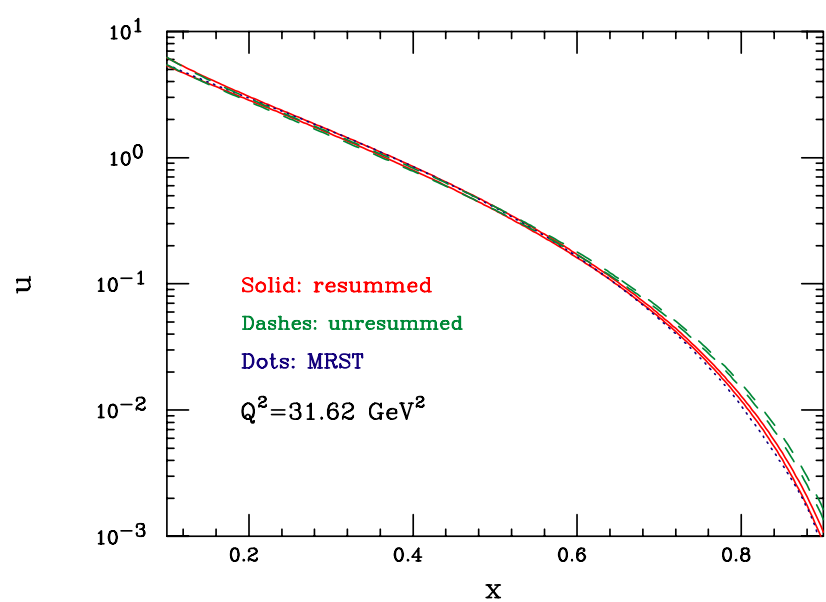

FIG. 7 (color online). As in Fig. 6, but at $Q^{2}=31.62 \mathrm{GeV}^{2}$.

resummation. Depletion of valence quarks at large $x$ is thus partly compensated by an increase at smaller values of $x$. The further change in sign at values of $x$ around 0.1 , on the other hand, cannot be taken too seriously, since it happens in a region which is dominated by extrapolation within our
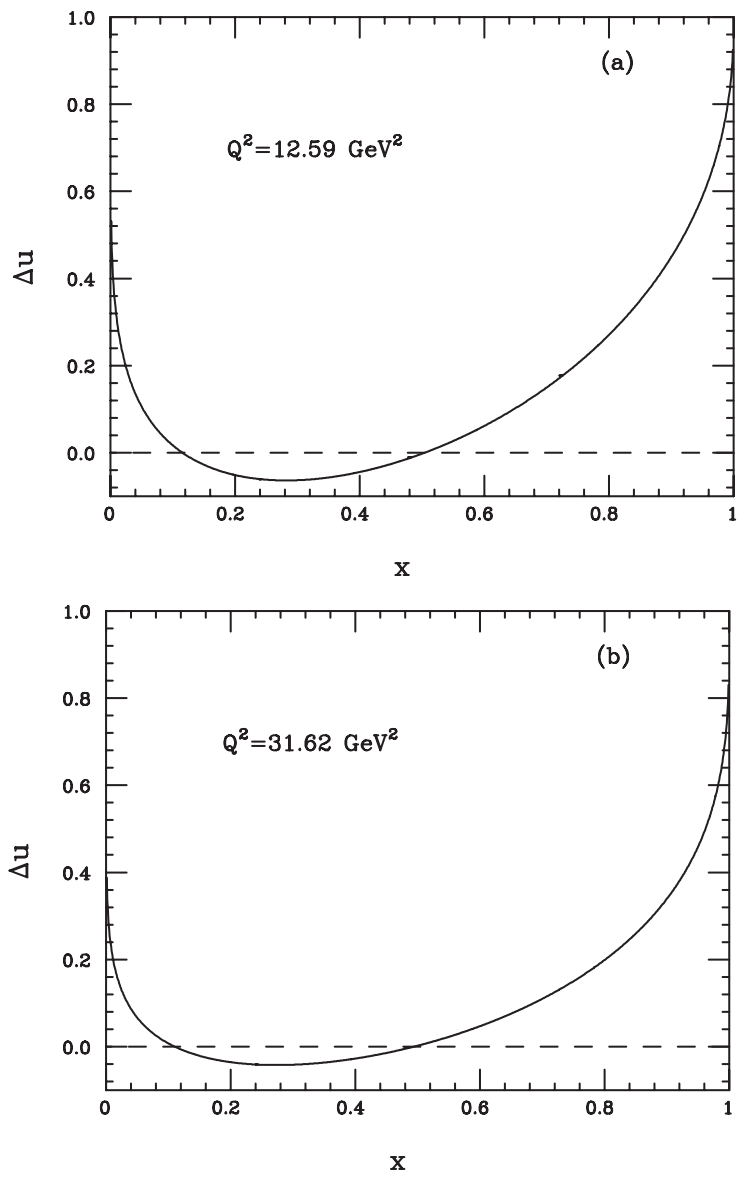

FIG. 8. Central value for the relative change in the up quark distribution, $\Delta u(x) \equiv\left(u_{\mathrm{NLO}}(x)-u_{\mathrm{res}}(x)\right) / u_{\mathrm{NLO}}(x)$, at $Q^{2}=$ 12.59 (a) and $31.62 \mathrm{GeV}^{2}$ (b). 


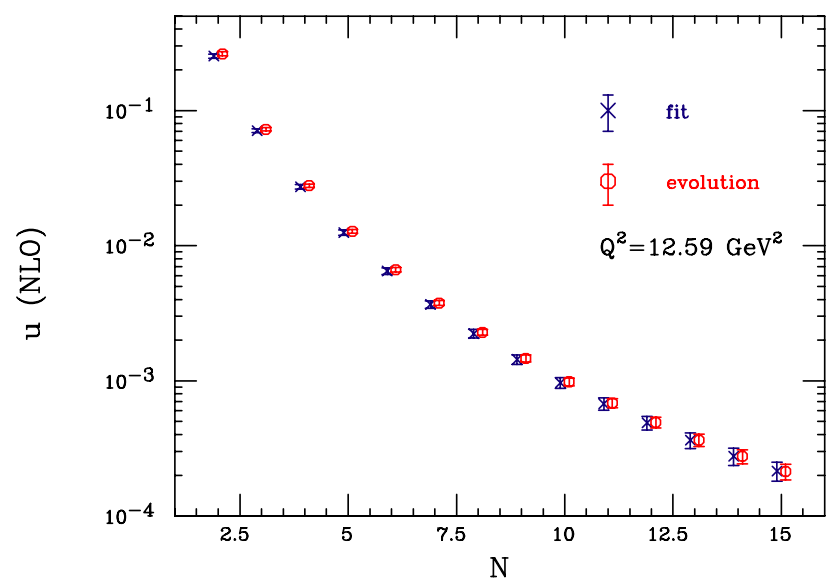

FIG. 9 (color online). Comparison of fitted moments of the NLO up quark distribution, at $Q^{2}=12.59 \mathrm{GeV}^{2}$, with moments obtained via NLO evolution from $Q^{2}=31.62 \mathrm{GeV}^{2}$.

current data set, so that errors are correspondingly very large. The impact of resummation is larger, as must be expected, at the lower value of $Q^{2}$.

At $Q^{2}=12.59 \mathrm{GeV}^{2}$ and large $x$, it is to be expected that power corrections will play a role too. We have chosen, however, not to introduce them explicitly in our parametrization of $F_{i}\left(x, Q^{2}\right)$ since, as discussed in the introduction, their effect is inevitably tied to the precise treatment of the resummation. Disentangling resummations and power corrections is best left to a more precise quantitative analysis performed in the context of a global fit. We have, in any case, checked that target mass corrections do not significantly influence our results.

We have verified that our fits, which are performed independently at two fixed values of $Q^{2}$, are consistent with perturbative evolution. To this end, we have evolved the moments of the up quark distribution, starting at $Q^{2}=$

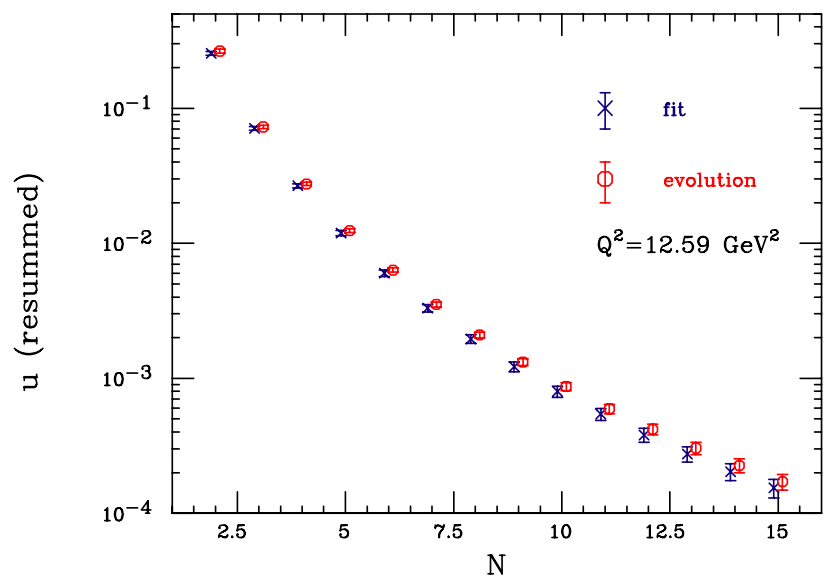

FIG. 10 (color online). As in Fig. 9, but comparing NLLresummed moments of the up quark density.
$31.62 \mathrm{GeV}^{2}$, using the NLO Altarelli-Parisi anomalous dimension, down to $Q^{2}=12.59 \mathrm{GeV}^{2}$, and we have compared the results of the evolution with the direct fits of Figs. 4 and 5. The comparison is shown in Figs. 9 and 10. The results are fully compatible within errors, and we believe that the agreement could be further improved if one included power corrections, which are relevant especially at $Q^{2}=12.59 \mathrm{GeV}^{2}$. In the evolution, we have used $\alpha_{s}\left(12.59 \mathrm{GeV}^{2}\right)=0.2394, \quad$ corresponding to $\alpha_{s}\left(31.62 \mathrm{GeV}^{2}\right)=0.2064$, or to $\Lambda_{\mathrm{QCD}}^{(5)}=226 \mathrm{MeV}$, with appropriate matching at the $b$-quark mass threshold, set to $m_{b}=4.5 \mathrm{GeV}$. Our choices are consistent with Ref. [2].

From a phenomenological point of view, we see that the impact of soft-gluon resummation on quark distributions can be sizeable, albeit only at values of $x$ which are quite large, say $0.55<x<0.75$. More precisely, as one can verify from Fig. 8, the suppression of the resummed up quark distribution with respect to the NLO one reaches $5 \%$ at $x \simeq 0.58,10 \%$ at $x \simeq 0.65$, and $20 \%$ at $x \simeq 0.75$ for $Q^{2}=12.59 \mathrm{GeV}^{2}$, while for $Q^{2}=31.62 \mathrm{GeV}^{2}$ the same suppression factors are reached at $x \simeq 0.61, x \simeq 0.69$, and $x \simeq 0.8$, respectively. Such values of $x$ can be relevant for several high energy processes, ranging from the production of high-mass Drell-Yan pairs, to high- $E_{T}$ jets, to the exchange of heavy resonances in the $t$-channel of hadron collisions. Considering, for example, the eccess of high- $E_{T}$ jets seen at the Tevatron Run I by CDF [41], the effect of including resummations in a PDF fit would have been to actually enhance the discrepancy between theory and experiment, since resummations suppress valence quarks at large $x$, and thus would have lowered the QCD prediction. Of course, a fully consistent treatment would have required making use of a resummed partonic cross section as well, which might have had a balancing effect. Interestingly, resummation may well be moving valence quarks from large to medium values of $x$, though the evidence for that in our present fit is at best qualitative. If that were the case, one might encounter several competing effects, depending on the partonic subprocess. For example, in Drell-Yan production at high mass and high rapidity, the heavy vector boson is produced by fusion of high- $x$ and low- $x$ partons, and one could have a depletion when the high- $x$ parton is a quark, or a slight enhancement (or no effect at all) when the high- $x$ parton is a gluon and the quark has lower momentum fraction. Finally, it should be noted that, in a fully consistent treatment, the depletion of the cross section due to resummed PDF's may well compete against the enhancement of the hard partonic cross section which is commonly found when resumming logarithms to that accuracy, resulting in a reduced impact of soft gluons at hadron level.

\section{OUTLOOK}

We have performed a first attempt to assess the impact of soft-gluon resummation on fits of parton distribution func- 
tions. We have argued that a global fit including soft-gluon effects is both feasible and desirable, from a theoretical as well as phenomenological point of view. That being said, it is useful to gauge the size of the effect that resummation might have. To that end, we have performed a simple fit of large- $x$ DIS data from the NuTeV, NMC, and BCDMS Collaborations. Our fit is not meant to be used as a complement or a substitute for a global fit: it is based on a small set of data, concentrated at large $x$, and does not consistently include all the constraints arising from sum rules and evolution which are properly taken into account in global fits. Our results should instead be seen as a first semiquantitative study of the impact of soft-gluon effects, and we believe that they might be an incentive for the dedicated collaborations performing PDF fits to include these effects in their algorithms. We have shown that, in the $\overline{\mathrm{MS}}$ scheme, the main effect of soft resummation is to suppress valence quark distributions at large $x$, by an amount ranging from a few percent to as much as $20 \%$, in the range $0.55<x<$ 0.75 . This suppression may be partly compensated by a weaker enhancement, of the order of a few percent, at medium values of $x, 0.1<x<0.5$, an effect which however falls largely inside our current statistical errors. It should be noted that a sizeable effect of this kind at large values of $x$ and moderate $Q^{2}$ will feed through to smaller values of $x$ at large $Q^{2}$ via evolution. We expect that including resummations should help to lower the theoretical uncertainty in the determination of PDF's, and more in general in QCD cross sections, both by reducing scale uncertainties, and by allowing for stronger constraints on large- $x$ partons, thanks to the inclusion of more data points. We note also that the effect of resummations on PDF's (a suppression) may turn out to be competing with the effect on partonic cross sections (in general, an enhancement). Disentangling such competing effects to gain a precise quantitative understanding of soft-gluon effects on hadron-level cross sections can only be achieved with a consistent treatment of resummations, including their effects both in PDF global fits and in hard cross sections.

\section{ACKNOWLEDGMENTS}

The authors would like to thank S. Forte, M. L. Mangano and M.H. Seymour for useful discussions, and L. Del Debbio, J. Rojo, and A. Piccione for help with the neural network code of the NNPDF Collaboration. We are also grateful to the NuTeV Collaboration, and especially to D. Naples and M. Tzanov, for their assistance in the use of the $\mathrm{NuTeV}$ structure function data. L. M. wishes to thank CERN for hospitality and support during the early phase of this work, which is also supported in part by MIUR under Contract No. 2004021808009.
[1] W. Giele et al., hep-ph/0204316.

[2] J. Pumplin, D. R. Stump, J. Huston, H. L. Lai, P. Nadolsky, and W. K. Tung, J. High Energy Phys. 07 (2002) 012.

[3] A. D. Martin, R. G. Roberts, W. J. Stirling, and R. S. Thorne, Phys. Lett. B 531, 216 (2002).

[4] S. Alekhin, Phys. Rev. D 68, 014002 (2003).

[5] A. Vogt, S. Moch, and J. A. M. Vermaseren, Nucl. Phys. B691, 129 (2004).

[6] S. Moch, J. A. M. Vermaseren, and A. Vogt, Nucl. Phys. B688, 101 (2004).

[7] G. Sterman, Nucl. Phys. B281, 310 (1987).

[8] S. Catani and L. Trentadue, Nucl. Phys. B327, 323 (1989).

[9] A. Banfi, G. P. Salam, and G. Zanderighi, J. High Energy Phys. 03 (2005) 073.

[10] A.D. Martin, R. G. Roberts, W. J. Stirling, and R. S. Thorne, Phys. Lett. B 443, 301 (1998).

[11] E. Gardi and R.G. Roberts, Nucl. Phys. B653, 227 (2003).

[12] G. Sterman and W. Vogelsang, hep-ph/0002132.

[13] J.A.M. Vermaseren, A. Vogt, and S. Moch, hep-ph/ 0504242.

[14] A. Vogt, Phys. Lett. B 497, 228 (2001).

[15] E. Gardi, G. P. Korchemsky, D. A. Ross, and S. Tafat, Nucl. Phys. B636, 385 (2002).

[16] T. O. Eynck, E. Laenen, and L. Magnea, J. High Energy Phys. 06 (2003) 057.
[17] C. Anastasiou, L. J. Dixon, K. Melnikov, and F. Petriello, Phys. Rev. Lett. 91, 182002 (2003).

[18] S. Alekhin, in the Proceedings of the HERA-LHC Workshop, DESY, 2005 (to be published).

[19] S. Catani, M. L. Mangano, P. Nason, C. Oleari, and W. Vogelsang, J. High Energy Phys. 03 (1999) 025.

[20] E. Laenen, G. Sterman, and W. Vogelsang, Phys. Rev. Lett. 84, 4296 (2000).

[21] G. Sterman and W. Vogelsang, Phys. Rev. D 71, 014013 (2005).

[22] N. Kidonakis and G. Sterman, Nucl. Phys. B505, 321 (1997).

[23] N. Kidonakis and J.F. Owens, Phys. Rev. D 63, 054019 (2001).

[24] M. Dasgupta, Pramana 62, 675 (2004).

[25] S. Kuhlmann et al., Phys. Lett. B 476, 291 (2000).

[26] M. Tzanov et al. (NuTeV Collaboration), hep-ex/0306035.

[27] D. Naples et al. (NuTeV Collaboration), hep-ex/0307005.

[28] M. Arneodo et al. (New Muon Collaboration), Nucl. Phys. B483, 3 (1997).

[29] A. C. Benvenuti et al. (BCDMS Collaboration), Phys. Lett. B 223, 485 (1989).

[30] A.C. Benvenuti et al. (BCDMS Collaboration), Phys. Lett. B 237, 592 (1990).

[31] M. Osipenko et al. (CLAS Collaboration), Phys. Rev. D 67, 092001 (2003); M. Osipenko et al., Phys. Rev. D 71, 
054007 (2005).

[32] S. Forte, L. Garrido, J. I. Latorre, and A. Piccione, J. High Energy Phys. 05 (2002) 062.

[33] L. Del Debbio, S. Forte, J. I. Latorre, A. Piccione, and J. Rojo (NNPDF Collaboration), J. High Energy Phys. 03 (2005) 080.

[34] S. Forte, J. I. Latorre, L. Magnea, and A. Piccione, Nucl. Phys. B643, 477 (2002).

[35] S. Forte and L. Magnea, Phys. Lett. B 448, 295 (1999).

[36] W. Seligman, Ph.D. thesis, Columbia University [Institution Report No. Nevis-292, 1997].
[37] S. Catani, B. R. Webber, and G. Marchesini, Nucl. Phys. B349, 635 (1991).

[38] G. Corcella and A. D. Mitov, Nucl. Phys. B676, 346 (2004).

[39] E. Laenen and S. O. Moch, Phys. Rev. D 59, 034027 (1999).

[40] S. Forte and G. Ridolfi, Nucl. Phys. B650, 229 (2003).

[41] T. Affolder et al. (CDF Collaboration), Phys. Rev. D 64, 032001 (2001); 65, 039903E (2002). 